\title{
Estimation d'une courbe de tarage hauteur-dénivelée-débit pour une rivière influencée par la marée
}

\author{
Benoît CAMENEN ${ }^{1}$, Guillaume DRAMAIS ${ }^{1}$, Jérôme LE COZ1 ${ }^{1}$, Tuan Duc HO², Nicolas GRATIOT², Stéphane PINEY ${ }^{3}$ \\ ${ }^{I}$ Irstea, UR HHLY, centre de Lyon-Villeurbanne, 5 rue de la Doua, BP 32108, F-69616 Villeurbanne Cedex, France benoit.camenen@irstea.fr \\ ${ }^{2}$ Care, IRD, Block B7, 268 Ly Thuong Kiet St, Dist. 10, Ho-Chi-Minh-Ville, Vietnam \\ ${ }^{3}$ DREAL Normandie, SRN/B2HPC, 1 rue Dufay, 76100 Rouen, France
}

\begin{abstract}
RÉSUMÉ. - Un modèle de courbe de tarage à deux points de mesure du niveau d'eau est proposé pour les rivières tidales. Il est basé sur une simple application de la loi de Manning-Strickler en se donnant la possibilité d'une pente négative pour obtenir un débit remontant la rivière. Deux variables permettent le calage du modèle : le coefficient de Strickler et le décalage temporel entre la pente mesurée par les deux niveaux et celle à appliquer à la section de référence. Le modèle a été appliqué sur la Rivière de Saïgon au Vietnam et la Seine en France où des campagnes de jaugeages $\mathrm{ADCP}$ ont été réalisées sur un ou deux cycles de marée. Les résultats sont très encourageants dans le cas de la Rivière de Saïgon où les variations de hauteur restent modérées. Dans le cas de la Seine, les résultats ne sont pas aussi satisfaisants du fait de la variation brutale du niveau d'eau et du débit lors du flot observée pendant une très forte marée qui rend le calage difficile. Le modèle semble néanmoins valide pour les rivières tidales tant que le gradient temporel du débit lors du flot de la marée reste modéré.
\end{abstract}

Mots-clés : Courbe de tarage, débit, marée, hydrométrie

\section{Estimation of a water level - discharge rating curve for a river influenced by the tide}

\begin{abstract}
A simple rating curve model using two water level measurements is proposed for tidal rivers. It is based on the Manning-Strickler equation adapted for possible negative slopes resulting from a negative discharge (upstream directed). The model calibration can be achieved thanks to two parameters only: the Strickler coefficient and the temporal lag between the measured slope and the slope to be applied at the reference section. The model has been applied to two tidal rivers for which ADCP gaugings have been achieved during two tidal cycles: the Saigon River in Vietnam and the Seine River in France. Results are encouraging for the Saigon River where water level variations remain mild. For the Seine River case, results are not as good due to a sharp variation of the water level and discharge during the flow tide that makes the calibration difficult. Nevertheless, the proposed model appears to be valid for tidal rivers as soon as the discharge temporal gradient during the tide flow remains moderate.
\end{abstract}

Key-words: Rating curve, water discharge, tide, hydrometry

\section{INTRODUCTION}

La connaissance des débits en continu reste un challenge pour mieux estimer la dynamique d'un bassin versant et prédire les risques d'inondation. Dans la majorité des cas, le débit est estimé indirectement à partir d'une ou plusieurs mesures de niveau d'eau et éventuellement une mesure locale de vitesse via une courbe de tarage [Rantz et al, 1982]. Dans le cas des rivières tidales, le niveau d'eau et le débit à une station de mesure peuvent être fortement influencés par la marée, ce qui rend ainsi l'estimation du débit par des méthodes classiques beaucoup plus difficile. Il y a de plus une forte interaction entre le débit entrant de la rivière et la marée qui se traduit principalement par l'atténuation de la marée [Cai et al, 2014] et l'augmentation du frottement moyen [Buschmann et al., 2009; Sassi \& Hoitink, 2013]. El Jabi et al. [1992] ont proposé une analyse statistique permettant de relier le niveau d'eau au débit et à la marée pour deux rivières canadiennes : la Saint-Jean (Nouveau Brunswick) et le Saint-Laurent (Québec). De façon similaire, des modèles analytiques ont récemment été proposés pour estimer le débit à partir d'une mesure de niveau d'eau et de la connaissance des caractéristiques de la marée [Moftakhari et al., 2013; Sassi \& Hoitink, 2013; Cai et al., 2014]. En parallèle, des courbes de tarage complexes à plusieurs variables d'entrée ont été calibrées à partir de méthodes se basant sur les réseaux de neurones [Habib \& Meselhe, 2006; Hidayat et al., 2014]. Ces méthodologies restent cependant difficilement applicables en mode opérationnel et présentent des incertitudes non négligeables. Plus récemment avec le développement des méthodes hydro-acoustiques, une mesure en continu a été rendue possible avec l'utilisation des cordes de vitesse [Simpson \& Bland, 2000], d'ADCP positionnés verticalement en fixe [Zhao et al., 2016] et des H-ADCP [Buschmann et al., 2009; Hoitink et al., 2009; Sassi et al., 2011]. Ces dernières méthodes restent aussi partielles et nécessitent un calage pour obtenir le débit total, en général en passant par la méthode de la vitesse témoin [ISO2425, 2010; Chen et al., 2012]. De plus, leur coût d'investissement et d'entretien reste encore prohibitif.

En France, le SCHAPI (service central d'hydrométéorologie et d'appui à la prévision des inondations) connaît ainsi 
des difficultés toutes particulières pour estimer le débit de ces rivières côtières et donc le risque d'inondation en cas de concomitance de la crue avec une forte marée. On peut retenir par exemple les polémiques relatives aux difficultés de prédiction des niveaux d'eau sur la Laïta à Quimperlé lors des inondations de janvier et février 2014 suite au passage de plusieurs tempêtes. Même si la prévision des crues ne s'appuie pas uniquement sur les courbes de tarage mais aussi sur une anticipation de l'évolution du niveau d'eau par une analyse hydro-météorologique complexe, l'amélioration de l'estimation des débits pour les rivières tidales est un prérequis pour l'amélioration des prévisions de crues. Actuellement, le risque inondation reste souvent étudié à partir d'abaques reliant la hauteur au point d'intérêt dans la zone sous influence de la marée au débit de la rivière avant influence marée et au coefficient de marée. De façon étonnante, on ne retrouve dans la littérature aucune application de méthodes simples à deux points de mesure à des rivières influencées par la marée alors même que cette méthode est couramment utilisée pour des stations à influence aval variable en amont de confluences ou barrages par exemple [Callède et al., 2001]. Il n'existe pas ou peu de courbes de tarage pour les rivières tidales basées sur un nombre restreint de points de mesure. Nous proposons donc ici d'appliquer un modèle simple à deux points de mesure, issu de l'application de l'équation de Manning-Strickler, sur des rivières tidales. Ce type de méthode peut maintenant être calibré grâce à l'utilisation de profileur ADCP. Ce dernier permet en effet de jauger rapidement une rivière tidale et ainsi de décrire la variation temporelle du débit lors d'un ou plusieurs cycles de marée. Cela était beaucoup plus délicat à l'époque où les mesures étaient réalisées à l'aide de flotteurs et/ou de moulinets, comme lors de la campagne de mesure en Seine aval rapportée par Laval [1955]. Il existe donc aujourd'hui des jeux de données permettant le calage et la validation de modèles de courbe de tarage en site influencé par la marée.
Après une rapide description des sites de mesure (Rivière de Saïgon et Seine) et des données disponibles, nous présenterons le modèle de courbe de tarage avec une application sur ces deux sites contrastés.

\section{SITES DE MESURE ET DONNÉES ACQUISES}

Deux sites de mesure caractérisés par une forte influence de la marée ont été considérés pour réaliser cette étude : la Rivière de Saïgon (Vietnam) et la Seine (France). La localisation précise des sites est donnée en Figure 1.

\section{II.1. Rivière de Saïgon (Vietnam)}

Des mesures de débit ont été acquises sur la Rivière de Saïgon au niveau de Phu Cuong (Nord d'Ho-Chi-Minh-ville, cf. Figure 1a) à l'aide d'un ADCP Rio Grande $600 \mathrm{kHz}$ fixé sur le côté de l'embarcation motorisée et couplé à un GPS. Les mesures ont été réalisées sur deux cycles de marée entre le 13 et le 14 septembre 2016. La Rivière de Saïgon à cette section mesure environ $200 \mathrm{~m}$ de large pour une profondeur maximale de $15 \mathrm{~m}$. La section se situe sur un secteur rectiligne avec un pont situé environ $200 \mathrm{~m}$ en aval. Il est à noter que les piles du pont influencent fortement les vitesses en amont pour les débits négatifs (flot). Deux capteurs de pression de type DIVER ont été posés pendant la durée des expériences sur le site de mesure des débits à Phu Cuong et en aval à Bach Dang (centre-ville d'Ho-Chi-Minh-ville, cf. Figure 1.a). La position de ces capteurs n'a cependant pas pu être géoréférencée en altitude.

La Figure 2 présente les acquisitions obtenues sur la Rivière de Saïgon. Les mesures de hauteur d'eau (Figure 2a) montrent une forte asymétrie de la marée mais pas de variation brutale. On observe de plus que le capteur à Bach Dang (a)

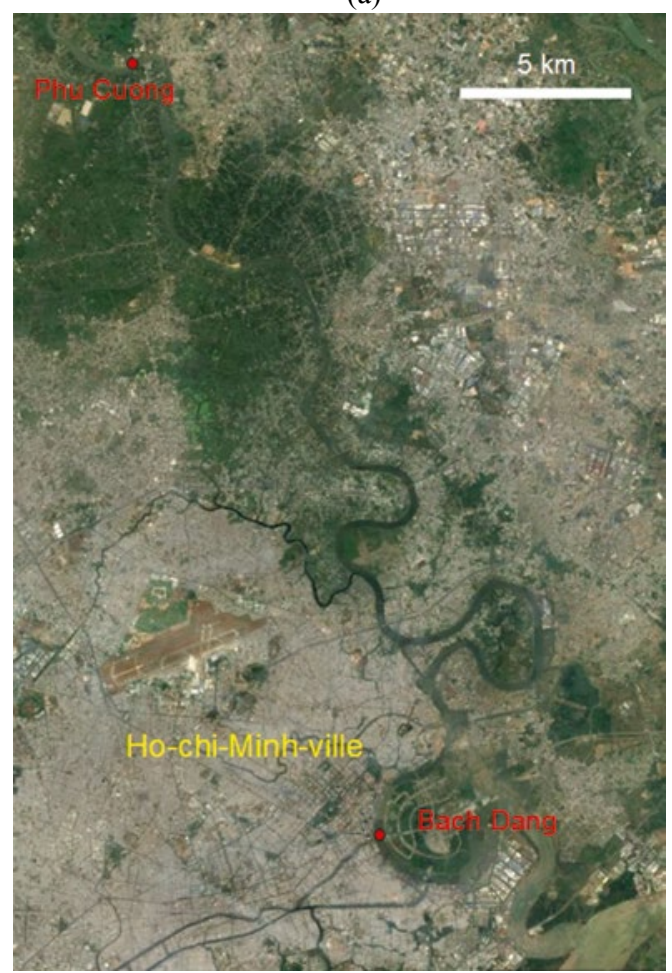

(b)
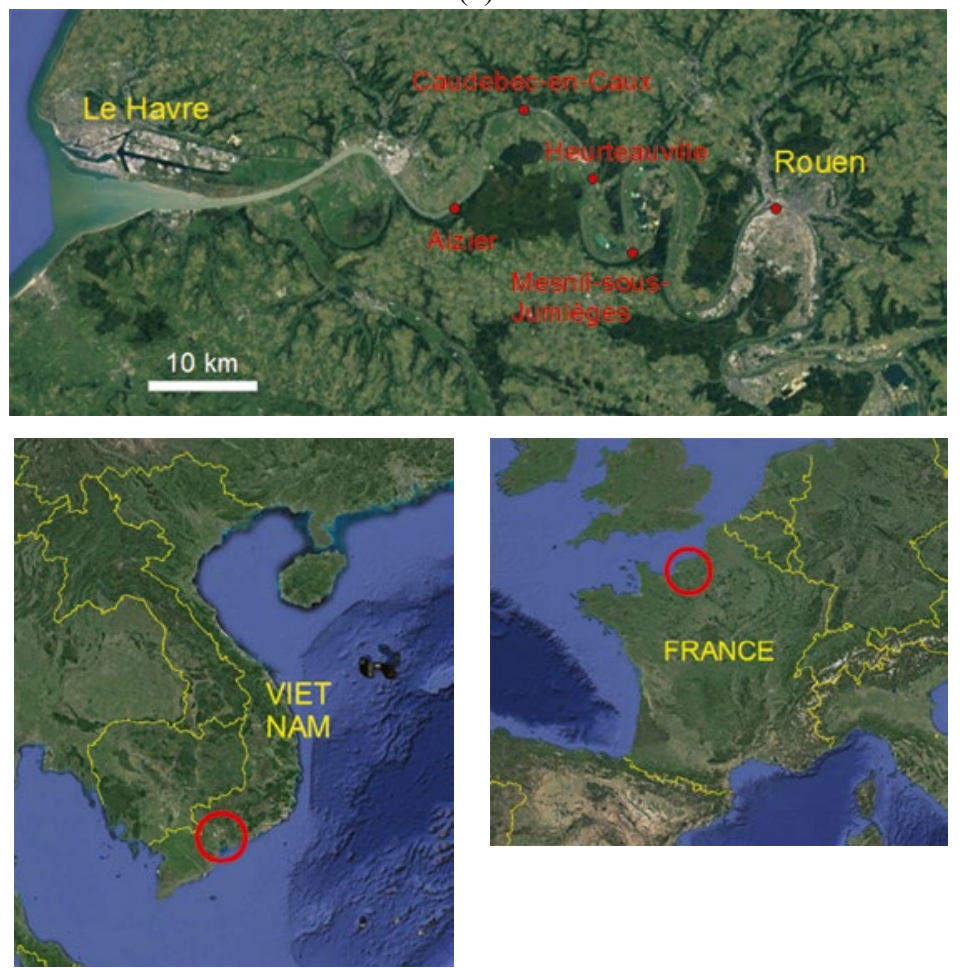

Figure 1 : Sites de mesure : la Rivière de Sä̈gon, Vietnam (a) et la Seine, France (b). Les limites d'influence de la marée se situent respectivement au lac Dau Tieng (environ $40 \mathrm{~km}$ en amont de Phu Cuong) et au barrage de Poses en amont de Rouen pour la Seine. 
(a)

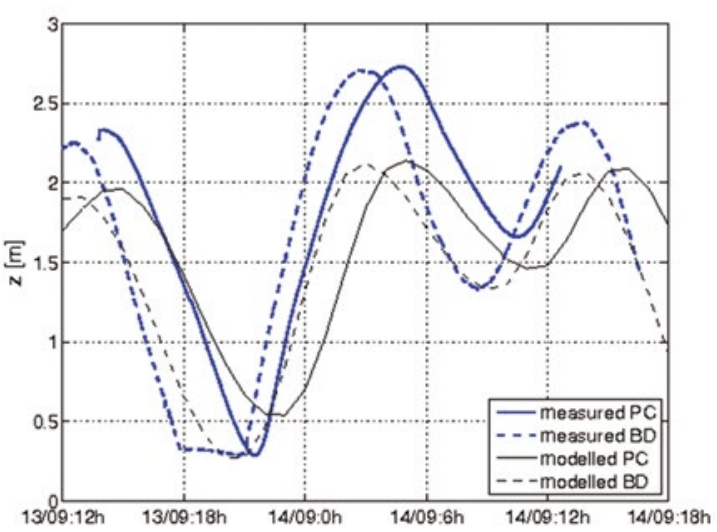

(b)

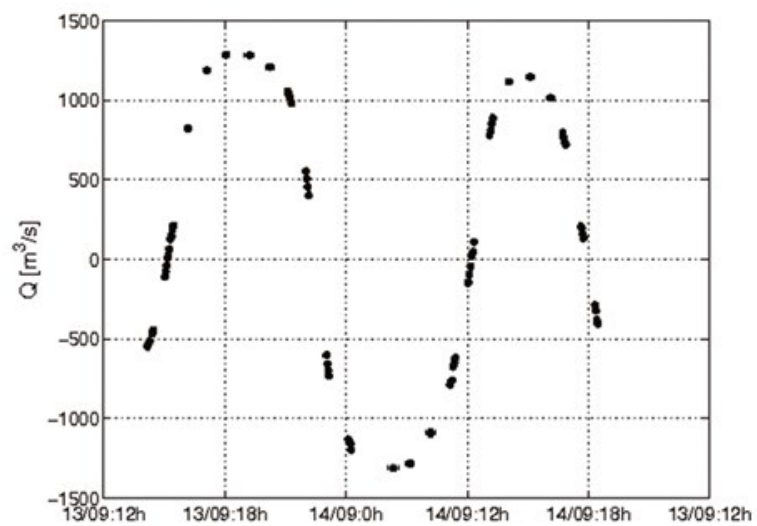

Figure 2 : Niveaux d'eau mesurés et modélisés sur la Rivière de Sä̈gon à Phu Cuong (PC) et Bach Dang (BD) (a) et débits mesurés par ADCP à Phu Cuong (b) lors de la campagne des 13 et 14 septembre 2016.

est passé hors d'eau le 13 septembre de $18 \mathrm{~h}$ à $21 \mathrm{~h}$. Sur cette même figure sont tracées les variations du niveau d'eau modélisées par les services hydrométriques du Vietnam. On peut s'apercevoir que l'amplitude de la marée n'est pas très bien reproduite (propagation de la marée modélisée sans connaissance du débit de la Rivière de Saïgon) ; un léger déphasage temporel, de l'ordre de $1 \mathrm{~h}$, peut aussi être observé.

Les jaugeages réalisés par ADCP sont présentés en Figure 2b. 103 traversées ont été réalisées lors de cette campagne de mesure pour 22 séries de quatre à six traversées toutes les heures environ. Le temps de traversée était de 5 min environ. La qualité du site a permis de valider certains jaugeages sur une unique traversée permettant ainsi d'observer en temps réel l'inversion de l'écoulement. Au final, 70 jaugeages sont conservés sur la Figure $2 b$ avec une incertitude estimée à $\pm 5 \%$. Ainsi cette campagne de mesure montre une variation des débits régulière sans variation brutale pendant le flot ou le jusant.

\section{II.2. Seine (France)}

Les sites de mesure sur la Seine (Rouen, Heurteauville, et Aizier d'amont en aval, cf. Figure 3.b) se situent systématiquement au droit d'un marégraphe du GPMR (Grand Port Maritime de Rouen) permettant ainsi de disposer de données de hauteurs exprimées, dans le même système altimétrique, en cotes marines du Havre $(\mathrm{m} \mathrm{CMH}=\mathrm{m}$ IGN69 + 4,378 $\mathrm{m})$ au pas de temps de 5 min, ce qui permet de connaître au droit de chaque section de jaugeage les variations correspondantes de la ligne d'eau. Il a été de plus choisi de faire la campagne de mesure un jour de grande marée : le 29 septembre 2015 (coefficient de marée de 117). Les jaugeages ont été réalisés sur chacun des sites avec deux ADCPs quasi tous couplés à des GPS, montés à bâbord et tribord du bateau, soit sur potence, soit sur support flottant, avec des fréquences d'émission différentes $(600$ et $1200 \mathrm{kHz})$ afin d'éviter toute interférence [Piney et al., 2017]. La Seine sur ces trois sites présente une largeur de 200 à $400 \mathrm{~m}$, impliquant des temps de traversée de près de 10 min qui ne permettent pas de faire plusieurs traversées pour valider un débit.

La Figure 3 présente les acquisitions obtenues sur la Seine le 29 septembre 2015. A la différence de la Rivière de Saïgon, on observe ainsi une variation brutale du niveau d'eau à la bascule au flot, impliquant une diminution très rapide des débits. Ainsi à Heurteauville, un très fort gradient de débit peut être observé ; ce dernier passant de +2400 à $-1500 \mathrm{~m}^{3} / \mathrm{s}$ en $10 \mathrm{mn}$ (Figure $3 b$ ). Pour ces jaugeages, la mesure de débit par ADCP est donc entachée d'une forte incertitude, compte-tenu de la durée de la traversée. On peut s'apercevoir également que les variations de débit sont fortement atténuées à Rouen alors que l'amplitude de la marée n'est plus que de 3,75 m (contre 4,8 m et $6,0 \mathrm{~m}$ à Heurteauville et Aizier respectivement). (a)

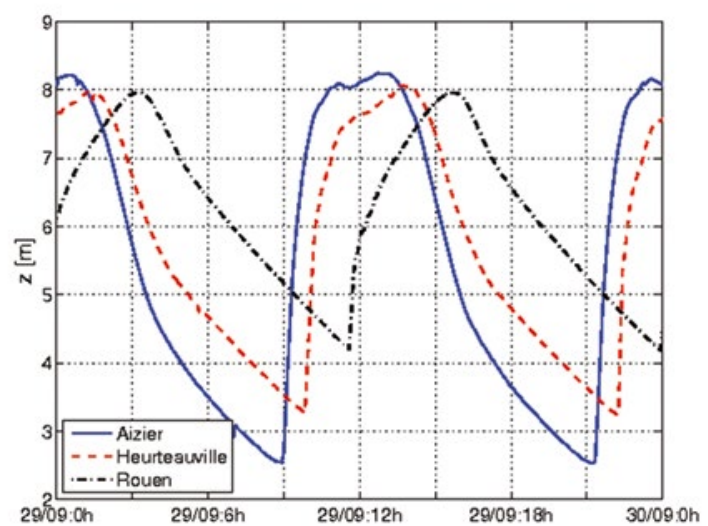

(b)

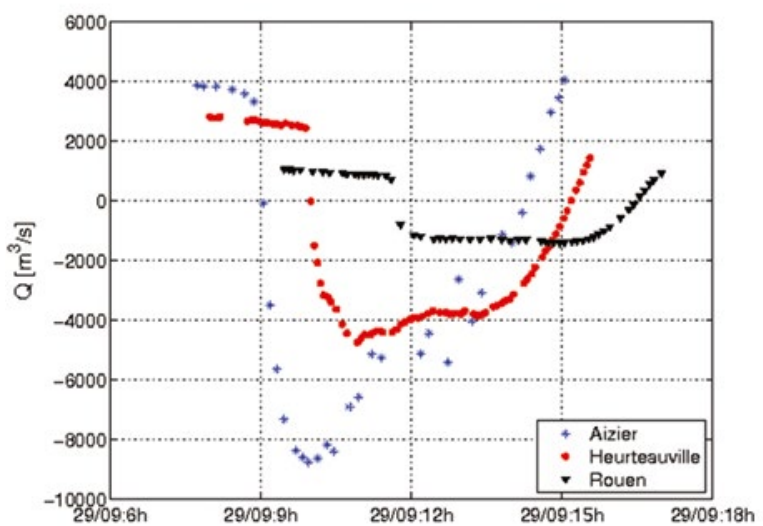

Figure 3 : Niveaux d'eau (a) et débits (b) mesurés sur la Seine à Aizier, Heurteauville et Rouen le 29 septembre 2015. 


\section{MODÈLES DE COURBE DE TARAGE HAUTEUR-DÉNIVELÉE-DÉBIT}

\section{III.1. Application de la loi de Manning-Strickler}

En faisant l'hypothèse d'un écoulement uniforme sur un tronçon prismatique, il est possible d'appliquer la formule de Manning-Strickler, y compris pour une rivière influencée par la marée si l'on suppose l'écoulement quasi-permanent.

$$
Q=K A_{w} R_{h}^{2 / 3} \sqrt{S}
$$

(Équation 1)

où $Q$ est le débit, $K$ le coefficient de Strickler, $A_{w}$ la section mouillée, $R_{h}$ le rayon hydraulique, et $S$ la pente de l'écoulement. En pratique, il est donc nécessaire pour estimer la pente de l'écoulement d'avoir deux points de mesure du niveau d'eau, $z_{1}$ et $z_{2}$, séparés d'une distance $L$ suffisamment grande pour que le dénivelé soit mesurable correctement. La pente de l'écoulement peut aussi s'inverser, et donc, comme indiqué Figure 2 et Figure 3, un écoulement remontant la rivière peut être observé (débit négatif, période de flot). En supposant $A_{w}=W\left(z_{1}-z_{0}\right)$ avec $W$ la largeur moyenne de la rivière sur le tronçon étudié et $z_{0}$ une hauteur de référence du fond, on obtient ainsi pour une rivière large où $R_{h} \sim h \sim z_{1}-z_{0}$ la courbe de tarage pour une station influencée par la marée :

$$
Q=\frac{\left(z_{1}-z_{2}+\Delta \mathrm{z}\right)}{\left|z_{1}-z_{2}+\Delta \mathrm{z}\right|} K W\left(z_{1}-z_{0}\right)^{5 / 3} \sqrt{\frac{\left|z_{1}-z_{2}+\Delta \mathrm{z}\right|}{L}},(\text { Équation 2) }
$$

avec $\Delta z$ une correction au cas où les niveaux $z_{1}$ et $z_{2}$ ne sont pas pris exactement dans le même repère altimétrique. Une difficulté apparaissant dans l'équation 2 est que la pente ne correspond pas exactement à la pente au point de mesure $z_{1}$ mais à une pente moyenne du tronçon I1-I2, soit à une pente d'un point légèrement situé en aval si le point $\mathrm{I} 2$ est situé en aval du point I1. L'onde de marée progressant vers l'amont, ce décalage spatial peut se traduire par un décalage temporel positif :

$$
Q(t)=\frac{S(t+\Delta \mathrm{t})}{|S(t+\Delta \mathrm{t})|} K W\left(z_{1}(t)-z_{0}\right)^{5 / 3} \sqrt{S(t+\Delta \mathrm{t})},(\text { Équation 3) }
$$

La prise en compte de ce décalage temporel peut être réalisée en première approximation en appliquant directement l'équation 2 avec le décalage temporel. Cependant, une erreur est alors introduite car ce décalage temporel ne doit pas s'appliquer à $z_{1}(t)$. Il est à noter de plus qu'une sous-estimation de la pente peut être observée dans le cas d'un front d'onde raide ne pouvant être décrit par des points I1 et 12 trop éloignés.

\section{III.2. Effet d'hystérésis dû à un régime transitoire}

Comme observé sur la Seine, l'onde de marée peut être très abrupte et donc, du fait du régime transitoire, peut avoir une influence non négligeable sur les débits. Pour un écoulement non-permanent, il est possible d'utiliser la correction de Jones [1916] pour la pente d'énergie :

$$
S=S_{n}+\frac{1}{C_{w}} \frac{\partial h}{\partial t}=\frac{z_{1}-z_{2}+" \mathrm{z}}{L}+\frac{1}{C_{w}} \frac{\partial h}{\partial t}
$$

(Équation 4)

où $C_{w}$ est la célérité de l'onde de marée (a priori négative) et $S_{n}$ la pente de l'écoulement pour un régime supposé quasi-permanent.

\section{APPLICATIONS}

\section{IV.1. Rivière de Saïgon (Vietnam)}

L'équation 2 a été appliquée pour le cas de la Rivière de Saïgon à Phu Cuong en prenant un coefficient de Strickler $K=26 \mathrm{~m}^{1 / 3} / \mathrm{s}$ (Figure 4). L'estimation du décalage temporel se fait aisément en comparant les résultats issus de l'équation 2 sans décalage temporel avec les données expérimentales ; une valeur $\Delta t=2 \mathrm{~h}$ a ainsi été obtenue. Les capteurs de niveau d'eau n'ayant pas été nivelés, le terme $\Delta z$ a été estimé afin de bien respecter l'inversion de l'écoulement. Les résultats du modèle sont particulièrement bons malgré une légère surestimation du débit lors du jusant. Cette surestimation disparait si l'on utilise l'équation 3 avec $K=28 \mathrm{~m}^{1 / 3} / \mathrm{s}$. Il est à noter que la légère sous-estimation du débit entre $20 \mathrm{~h}$ et $23 \mathrm{~h}$ le 13 septembre correspond à la période où le capteur aval était hors eau (cf. Figure $2 \mathrm{a}$ avec le décalage $\Delta t=2 \mathrm{~h}$ ), et correspond en réalité à une lacune de mesure.

\section{IV.2. Seine (France)}

De la même manière que pour la Rivière de Saïgon, nous avons appliqué l'équation 2 sur la Seine à Heurteauville. Du fait de la présence de plusieurs marégraphes en amont (Mesnil-sous-Jumièges, Rouen) et en aval (Caudebecen-Caux, Aizier), il est possible d'estimer la pente moyenne de l'écoulement à partir de ces différents points. Il apparaît que les résultats sont beaucoup moins bons que pour la Rivière de Saïgon et très sensibles au choix des points de mesure (Figure 5).

Les principales erreurs sont observables en fin de période de flot où des pics non-physiques sont modélisés et en fin du jusant où les débits augmentent beaucoup plus rapidement que les observations (et/ou avec un décalage temporel). Les coefficients de Strickler estimés varient entre $K=36 \mathrm{~m}^{1 / 3} / \mathrm{s}$ et $K=45 \mathrm{~m}^{1 / 3} / \mathrm{s}$ selon les points de mesure. Le décalage en temps est aussi difficile à caler sans doute du fait de la rapidité du changement de pente au flot et l'atténuation de la marée impliquant une pente non-uniforme sur le

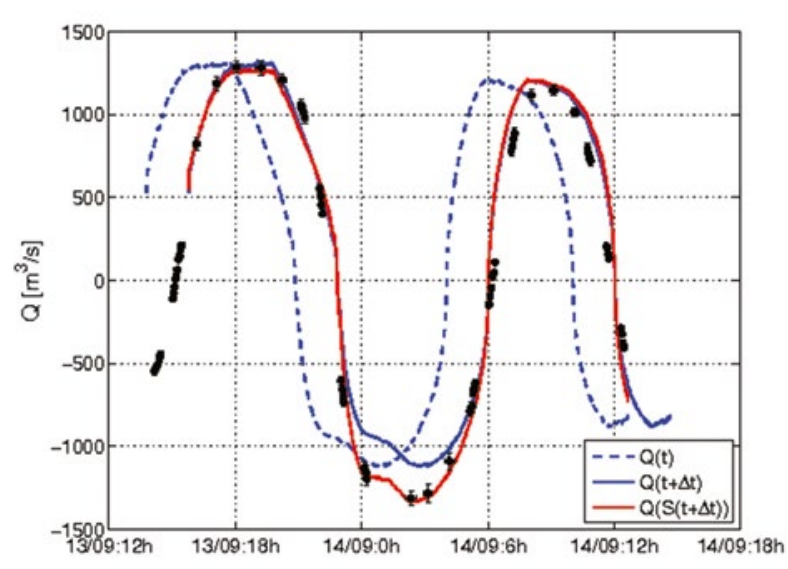

Figure 4 : Débit mesurés et modélisés à Phu Cuong les 13 et 14 septembre 2016. Les courbes bleues $Q(t)$ et $Q(t+t)$ correspondent à l'équation 2 avec $K=26 \mathrm{~m}^{1 / 3} / \mathrm{s}$ sans ou avec prise en compte d'un décalage temporel, respectivement, et la courbe rouge $Q(S(t+t))$ correspond à l'équation 3 avec $K=28 \mathrm{~m}^{1 / 3} / \mathrm{s}$. 
(a)

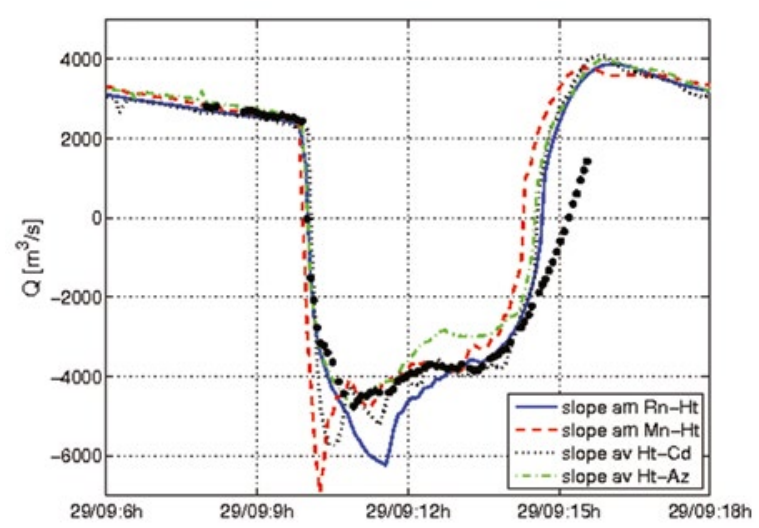

(b)

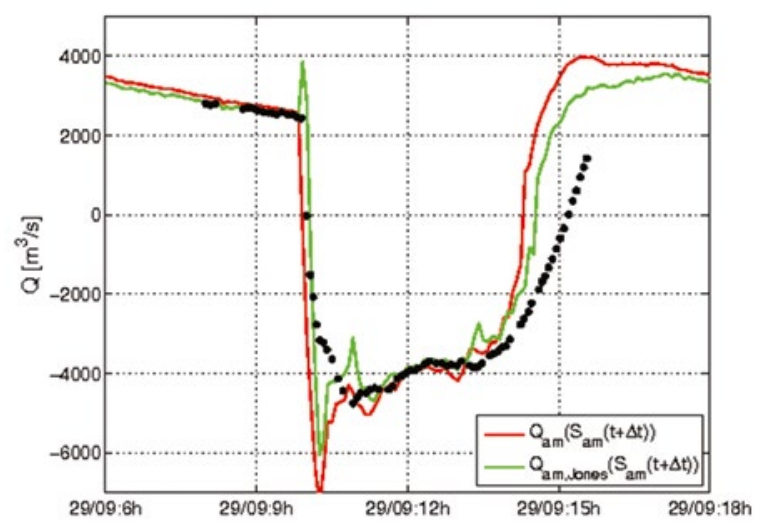

Figure 5 : Débits sur la Seine à Heurteauville mesurés et modélisés avec l'équation 2 pour différentes estimations de la pente (a) et modélisés avec l'équation 3 à partir de la pente entre Heurteauville et Mesnil-sous-Jumièges en utilisant ou non la formulation de Jones (b).

tronçon. Ceci peut expliquer les résultats sensiblement meilleurs si l'estimation de la pente est faite à partir des stations les plus proches (Mesnil-sous-Jumièges ou Caudebec-en-Caux). Il serait donc utile de tester le modèle sur un jour de plus faible marée pour vérifier si un calage est alors possible.

La correction de Jones pour l'écoulement transitoire est testée (cf. Figure 5b) avec une célérité de l'onde de marée estimée à $C_{w}=20 \mathrm{~m} / \mathrm{s}$. Si elle permet d'améliorer les résultats en fin du jusant, cela reste insuffisant. De plus, du fait du calcul d'un gradient de hauteur, des pics apparaissent à la bascule ( $\partial h / \partial t$ élevé) si l'on choisit une valeur de $C_{w}$ plus faible.

\section{CONCLUSION ET PERSPECTIVES}

Un modèle de courbe de tarage à deux points de mesure du niveau d'eau est proposé pour les rivières tidales. Il est basé sur une simple application de la loi de Manning-Strickler en se donnant la possibilité d'une pente négative pour obtenir un débit remontant la rivière. Le modèle a été appliqué sur deux campagnes de jaugeage ADCP réalisées sur la Rivière de Saïgon en septembre 2016 et sur la Seine en septembre 2015 lors d'une forte marée. Un simple calage du coefficient de Strickler, de l'écart de nivellement des deux points de mesure et du décalage temporel lié à la mesure de la pente sur la partie aval de la station a permis d'obtenir de très bons résultats dans le cas de la Rivière de Saïgon. Dans le cas de la Seine pour une forte marée, les résultats ne sont pas aussi satisfaisants du fait de la variation brutale du niveau d'eau et du débit lors du flot. L'application de la correction de Jones pour les écoulements non-stationnaires améliore légèrement les résultats mais reste insuffisante pour reproduire correctement les débits lors du jusant.

Le modèle de courbe de tarage à double échelle semble donc applicable aux rivières tidales pour des cas de marée ne montrant pas de variation abrupte du niveau d'eau lors du flot de la marée. Une analyse bayésienne permettra d'affiner le calage de la courbe de tarage avec une influence aval variable (coefficient de Strickler et décalage temporel) et de déterminer les incertitudes associées [Petersen-Overleir \& Reitan, 2009; Mansaranez et al., 2016]. Il reste à voir cependant s'il est possible d'améliorer le modèle proposé dans le cas de marée fortement asymétrique et brutale, l'amortissement de la marée pouvant varier selon le flot ou le jusant et affecter le frottement moyen de la rivière [Sassi \& Hoitink, 2013]. L'utilisation de modèles analytiques [Moftakhari et al., 2013; Sassi \& Hoitink, 2013; Cai et al, 2014] pourrait éventuellement permettre d'améliorer de façon simple notre modèle de courbe de tarage. Une courbe de tarage validée pour une rivière tidale aura un fort intérêt pour l'estimation du débit en continu, du débit net et des flux associés (matières en suspension et polluants associés ou dissous). Une telle courbe peut être très utile pour la navigation ou la production électrique (hydrolienne) du fait d'une meilleure prédiction des vitesses moyennes d'écoulement. Elle peut aussi avoir une grande utilité quant à la compréhension du risque d'inondation en cas de concomitance de forte marée et crue d'une rivière tidale car elle ne nécessite pas de connaissance préalable du débit amont et du coefficient de marée, ni des surcotes dues aux dépressions en mer.

\section{REMERCIEMENTS}

Les mesures expérimentales réalisées sur la Rivière de Saïgon ont été financées par la région Auvergne-Rhône-Alpes grâce au projet CMIRA Coopera Vietnam, Saigon : la ville et le fleuve, 2016-2017. L'organisation logistique de la mission a été menée par l'équipe du CARE à Ho Chi Minh ville avec le soutien du réseau RECIF et de l'IRD. Nous remercions aussi la DREAL Normandie, la DRIEE Ile de France et le Cerema de Blois pour la mise à disposition des données acquises sur la Seine. Enfin, nous remercions les relecteurs (A. Belleville et $\mathrm{S}$. Poligot-Pitsch) pour leurs commentaires et suggestions.

\section{REFERENCES}

Buschman F. A.,Hoitink A. J. F., Van Der Vegt M. \& Hoekstra P. (2009) - Subtidal water level variation controlled by river flow and tides. Water Resources Res, 45(W10420), 1-12.

Cai H., SaveniJe H. H. G. \& Jiang C. (2014) - Analytical approach for predicting fresh water discharge in an estuary based on tidal water level observations. Hydrol. Earth Syst. Sci. Discuss, 11, 7053-7087.

Callède J., Kosuth P. \& De Oliveira E. (2001) - Établissement de la relation hauteur-débit de l'Amazone à Óbidos: Méthode 
de la dénivelée normale à géométrie variable. Hydrological Sciences $J, \mathbf{4 6}, 451-463$.

Chen Y.-C., Yang T.-M., Hsu N.-S. \& Kuo T.-M. (2012) Real-time discharge measurement in tidal streams by an index velocity. Environ. Monit. Assess, 184, 6423-6436.

El Jabi N., Wakim G. \& SARRaF S. (1992) - Stage discharge relationship in tidal river. J. Waterways, Port, Coastal \& Ocean Eng, 118, 166-174.

Habib E. H. \& Meselhe E. A. (2006) - Stage-Discharge Relations for Low-Gradient Tidal Streams Using Data-Driven Models. J. Hydraulic Eng, 32(5), 482-492.

Hidayat H., Hoitink A. J. F., SAssi M. G. \& ToRfS P. J. J. F. (2014) - Prediction of Discharge in a Tidal River Using Artificial Neural Networks. J. Hydrologic Eng, 19(8, 04014006), 1-8.

Hoitink A. J. F., Buschman F. A. \& Vermeulen B. (2009) Continuous measurements of discharge from a horizontal acoustic Doppler current profiler in a tidal river. Water Resources Res, 45, 1-13.

ISO (2010) - Hydrometry - Measurement of liquid flow in open channels under tidal conditions. International Organization for Standardization; iso 2425.

JONES B. E. (1916) - A method of correcting river discharge for a changing stage. U. S. Geological Survey. Water Supply, Paper 375-E.

Laval D. (1955) - Mesures de débit faites en Seine-Maritime au cours de la crue de janvier 1955. Combinaison de la crue et de la marée. La Houille Blanche, A, 307-313.

Mansanarez V., Le Coz J., Renard B., Lang M., Pierrefeu G. \& VAuchel P. (2016) - Bayesian analysis of stage-fall-dis- charge rating curves and their uncertainties. Water Resources Res, 52, 7424-7443.

Moftakhari H. R., Jay D. A., Talke S. A., Kukulka T. \& BROMIRSKi P. D. (2013) - A novel approach to flow estimation in tidal rivers. Water Resources Res, 49, 4817-4832.

Petersen-Overleir A. \& Reitan T. (2009) - Bayesian analysis of stage-fall-discharge models for gauging stations affected by variable backwater. Hydrological Processes, 23, 3057-3074.

Piney S., Helouin S., Morel G., Hebrard A., Glaziou G., Diribarne J. \& S. Kopp (2017) - Mesures de débits en estuaire : l'exemple de la Seine. Congrès SHF : «Hydrométrie 2017, Lyon 14-15 mars 2017 ».

RANTZ S. E. AND Others (1982) - Measurement of Stage and Discharge. Paper 2175. Measurement and Computation of Streamflow : USGS, Water Supply, 1, 373 p.

SASsI M. G. \& Hoitink A. J. F. (2013) - River flow controls on tides and tide-mean water level profiles in a tidal freshwater river. J. Geophysical Res, 118, 4139-4151.

Sassi M. G., Hoitink A. J. F., Vermeulen B. \& Hidayat (2011) - Discharge estimation from H-ADCP measurements in a tidal river subject to sidewall effects and a mobile bed. Water Resources Res, 47(W06504), 1-14.

Simpson M. R. \& Bland R. (2000) - Methods for accurate estimation of net discharge in a tidal channel. IEEE J. Oceanic Eng, 25(4), 437-445.

Zhao J., Chen Z., Zhang H. \& Wang Z. (2016) - Multiprofile discharge estimation in the tidal reach of Yangtze Estuary.). J. Hydraulic Eng, 142(12, 04016056), 1-12. 